\title{
FORS spectroscopy of galaxies in the Hubble Deep Field-South $\star, \star \star$
}

\author{
D. Rigopoulou ${ }^{1,2}$, W. D. Vacca ${ }^{2,3}$, S. Berta ${ }^{4}$, A. Franceschini ${ }^{4}$, and H. Aussel ${ }^{5}$ \\ 1 Astrophysics, University of Oxford, Denys Wilkinson Building, Keble Road, Oxford OX1 3RH, UK \\ e-mail: dar@astro.ox.ac.uk \\ 2 Max-Planck-Institut für extraterrestrische Physik (MPE), Postfach 1312, Garching 85741, Germany \\ 3 USRA, NASA Ames Research Center, MS 144-2 Moffett Field, CA 94035-1000, USA \\ 4 Dipartimento di Astronomia, Universita' di Padova, Vicolo dell'Osservatorio 2, 35122 Padova, Italy \\ 5 Institute for Astronomy, 2680 Woodlawn Drive, Honolulu, Hawaii 96822, USA
}

Received 24 July 2003 / Accepted 18 December 2004

\begin{abstract}
We present low resolution multi-object spectroscopy of an $I$-band magnitude limited $\left(I_{\mathrm{AB}} \simeq 23-23.5\right)$ sample of galaxies located in an area centered on the Hubble Deep Field-South (HDFS). The observations were obtained using the Focal Reducer/low dispersion Spectrograph (FORS) on the ESO Very Large Telescope. Thirty-two primary spectroscopic targets in the HST-WFPC2 HDFS were supplemented with galaxies detected in the Infrared Space Observatory's survey of the HDFS and the ESO Imaging Deep Survey to comprise a sample of 100 galaxies for spectroscopic observations. Based on detections of several emission lines, such as [OII] $\lambda 3727, \mathrm{H}_{\beta}$ and [OIII] $\lambda 5007$, or of other spectroscopic features, we measured accurate redshifts for 50 objects in the central HDFS and flanking fields. The redshift range of the current sample of galaxies is 0.6-1.2, with a median redshift of 1.13 (at $I \simeq 23.5$ not corrected for completeness). The sample is dominated by starburst galaxies with only a small fraction of ellipticals $(\sim 10 \%)$. For the emission line objects, the extinction corrected [OII] $\lambda 3727$ line strengths yield estimates of star formation rates in the range 0.5-30 $M_{\odot} \mathrm{yr}^{-1}$. We used the present data to derive the [OII] $\lambda 3727$ luminosity function up to redshift of 1.2. When combined with [OII] $\lambda 3727$ luminosity densities for the local and high redshift Universe, our results confirm the steep rise in the star formation rate (SFR) to $z \simeq 1.3$.
\end{abstract}

Key words. cosmology: observations - surveys - galaxies: luminosity function, mass function - galaxies: starburst

\section{Introduction}

The Hubble Deep Field-South (HDFS, Williams et al. 2000; Casertano et al. 2000) has been the subject of extensive follow-up ground-based imaging observations at optical (ESO-Imaging Survey (EIS), DaCosta et al. 1998; Big Throughput Camera (BTC) survey, Teplitz et al. 2001), nearinfrared (e.g DaCosta et al. 1998; Vanzella et al. 2001; Rudnick et al. 2001; Saracco et al. 2001) and mid-infrared (Infrared Space Observatory (ISO): Oliver et al. 2002; Mann et al. 2002; Aussel et al. 2005, in prep.) wavelengths. The field has also been observed by the Spitzer Telescope (Huang et al. 2005, in prep.). The unprecedented spatial resolution and depth of the Hubble Space Telescope (HST) images have allowed a reliable morphological classification of objects in the field (e.g. the SUNY collaboration, available at http://www.ess. sunysb.edu/astro/hdfs/wfpc2/

\footnotetext{
* Figures 1 and 2 are only available in electronic form at http://www . edpsciences.org

$\star \star$ Tables 2 and 3 are only available in electronic form at the CDS via anonymous ftp to cdsarc.u-strasbg.fr $(130.79 .128 .5)$ or via http://cdsweb.u-strasbg.fr/cgi-bin/qcat?]/A+A/440/61
}

wfpc2.html). In addition, many groups (e.g. Vanzella et al. 2001; Rudnick et al. 2001) have used various techniques to estimate photometric redshifts for large samples of galaxies detected in the HDFS region. With such a wealth of imaging observations already in place, a systematic spectroscopic followup of objects detected in the HDFS is imperative in order to fully exploit the data. To date, only a limited number of redshifts have been determined for selected objects in the HDFS: Lyman break galaxy candidates by Cristiani et al. (2000) and Vanzella et al. (2002); ISO-detected objects by Rigopoulou et al. (2000) and Franceschini et al. (2003); objects in the vicinity of the quasar J2233-606 by Tresse et al. (1999) and Bergeron et al. (1999). Recently, Vanzella et al. (2002) reported on low resolution spectroscopy of a sample of 65 galaxies from the HDFS targetting primarily high-redshift $(z>2)$ candidates while Sawicki \& Mallen-Ornellas (2003) targeted lower redshift objects.

In this paper, we report on the optical spectroscopy of 100 objects located in the HDFS and flanking fields. We have determined accurate redshifts for 50 objects. The sample selection, observations and data reduction are explained in Sect. 2. The redshifts and redshift distribution are given in Sect. 3 while 
star formation rates and extinction estimates are discussed in Sect. 4. Finally, in Sect. 5 the [OII] $\lambda 3727$ luminosity function and the global star formation rate densities are presented. Throughout the paper we use $H_{0}=70 \mathrm{~km} \mathrm{~s}^{-1} \mathrm{Mpc}^{-3}, q_{0}=$ 0.5 unless otherwise stated.

\section{Sample selection, observations, and data reduction}

The WFPC2 observations of the HDFS resulted in the detection of a number of objects down to a limiting magnitude $F 814 W_{\mathrm{AB}} \sim 28 \mathrm{mag}$ (Casertano et al. 2000). From this sample, galaxies were selected with $F 814 W_{\mathrm{AB}}=23.7$ (corresponding to $I_{\text {Vega }}=23 \mathrm{mag}$ ), a limit imposed by the sensitivity of the FORS1 150I grism. This criterion resulted in a primary sample of 32 objects all within the central WFPC2 field. The primary sample was supplemented with objects from the Flanking Fields, all of which have identifications in the ESO Deep Imaging Survey (EIS) and the BTC catalog. Additionally we included objects selected from the ISO HDFS detections (Aussel et al. 2005, in prep.). In all, our final sample included 100 objects.

The spectroscopic observations were carried out as part of ESO program ID 65.O-0418(A) from August 24-28, 2000, using the Focal Reducer Spectrograph (FORS1) (Appenzeller 2000), in the Multi-Object-Spectroscopy (MOS) mode, on the ANTU-ESO telescope (formerly UT1), on Paranal, Chile. The data presented here have been retrieved from ESO's VLT science archive.

In the FORS1/MOS mode, 19 slits are placed on a mask covering a field of view $($ FOV) of $6.8 \times 6.8 \mathrm{sq}$ arcmin. To cover the 30 primary objects 5 MOS settings were needed. The supplemental objects were observed in the slits that were not occupied by the primary targets in each MOS setting. The slits were 1.2 wide and 22.5 arcsec long. In most cases the target galaxy was placed in the middle of the slit, so as to enable proper background substraction. In a few cases a second galaxy happened to fall in the same slit. Spectra of these serendipitous galaxies were also analysed. The GRIS-300V, and GRIS-150I grisms were used, covering the 4450-8650 and 6000-11000 $\AA$ ranges, respectively. The choice of grism was based on previous photometric redshift estimates for sample galaxies; for galaxies with $z_{\text {phot }}<0.7$ the GRIS-300V grism was used, while the GRIS-150I grism was used for those galaxies with $z_{\text {phot }}>0.7$. The pixel scale was 0.2 arcsec/pixel while the resolution was $\lambda / \Delta \lambda=440$ and 260 for the GRIS-300V and GRIS-150I grisms, respectively. At each MOS position, three exposures of $1900 \mathrm{~s}$ each were obtained, for a total integration time of $5700 \mathrm{~s}$ per position. The seeing during acquisition of the spectra varied between $0.8-1.5$ arcsec. In Table 1 we present details of the observations.

For each field there were 3 separate exposures which were co-added before spectral extraction. We reduced the MOS spectra by treating each of the 19 slits separately and using standard $\mathrm{IRAF}^{1}$ routines. The individual spectra were

\footnotetext{
${ }^{1}$ IRAF is distributed by the National Optical Astronomy Observatories, which are operated by the Association of Universities
}

Table 1. Log of the FORS1/MOS Observations.

\begin{tabular}{ccccccc}
\hline \hline Field & RA(J2000) & Dec(J2000) & Grism & Filter & $\lambda / \Delta \lambda$ & $t_{\exp }(\mathrm{s})$ \\
\hline M 2 & $22: 32: 54.5$ & $-60: 33: 45.9$ & $300 \mathrm{~V}$ & GG435 & 440 & 5700 \\
M 4 & $22: 33: 12.0$ & $-60: 32: 35.7$ & $300 \mathrm{~V}$ & GG435 & 440 & 5700 \\
M 5 & $22: 32: 43.9$ & $-60: 33: 24.7$ & $150 \mathrm{I}$ & GG435 & 260 & 5700 \\
M 9 & $22: 32: 39.4$ & $-60: 33: 57.0$ & $150 \mathrm{I}$ & OG590 & 260 & 5700 \\
M 10 & $22: 32: 59.6$ & $-60: 34: 18.1$ & 150I & GG435 & 260 & 5700 \\
\hline
\end{tabular}

bias-subtracted and flat-fielded in the standard manner. To remove the background sky emission we fit a low-order polynomial in the spatial direction at each column and then subtracted it for each individual exposure. Most of the cosmic ray hits in the sky region of the spectral images were excluded from the fits with a sigma-rejection algorithm. We then extracted a variance-weighted one-dimensional spectrum. The final spectrum was wavelength calibrated using arc lamp spectra and flux-calibrated using the spectro-photometric standard stars included in the MOS frame. The spectra of the standard stars were reduced in the same way as the galaxy spectra.

In Table 2 we present an inventory of all objects targeted with FORS1/MOS. We list galaxy number, coordinates (in those cases where two galaxies are included in the slit we report only the position of the sample galaxy) and optical (UBVRI) and near-infrared $(J H K)$ photometry. We give $A B$ magnitudes, taken mostly from EIS but supplemented with data from the BTC survey available from http://hires.gsfc.nasa.gov/ research/hdfs-btc. Additionally, we list HST identifications from the HDFS-WFPC2 catalogue (available from http://archive.stsci.edu/pub/hdf-south/version2) and the HDFS WFPC2 flanking fields catalogue (available from http://archive.stsci.edu/pub/hdf-south/ version1). Finally, we also give ISO identifications (from Aussel et al. 2005, in prep.).

\section{Results}

\subsection{Spectroscopic measures}

In Figs. 1a-1f and 2a-2b we show the final wavelength- and flux-calibrated spectra for each object in the M 2, M 4, M 5 , M 10 (Figs. 1a-1f) and M 9 fields (Figs. 2a-2b). The spectra have been grouped in the two figures according to the wavelength coverage: spectra in Fig. 1 cover the 4450 to $8200 \AA$ range (corresponding to the GRIS-300V grism) while those in Fig. 2 cover the 6000-9200 $\AA$ range (corresponding to the GRIS-150I grism). Redshifts were determined based on the wavelengths of the detected emission lines (usually the [OII] $\lambda 3727$ line originating in a starburst) or, in a few cases, absorption features such as the CaII H, K features (at 3968.5 and $3933.7 \AA$ ) prominent in the spectra of elliptical galaxies. When multiple emission lines were detected, we averaged the redshifts from these lines. In the cases where we detect absorption features we have not attempted to measure any

for Research in Astronomy, Inc. under the cooperative agreement with the National Science Foundation. 
properties of the feature. The shape of the underlying continuum (especially in cases where emission lines were present) was also taken into account and helped to constrain the redshifts more efficiently. In each spectrum we report the galaxy number (from Table 2) and the corresponding redshift and mark the position of both detected and expected emission/absorption lines. Note that emission features are not always seen at the expected locations of some lines (e.g., H $\beta$ or [OIII] $\lambda 5007$ ). In several spectra there are residual [O I] night-sky lines at $\lambda \lambda 5577$ and 6300-6364 that can sometimes lead to "spurious" emission features in the spectra. These lines are highly variable and could not always be properly removed during the sky subtraction step.

In total we have obtained spectra for 100 objects. Some of the objects were determined to be stars. We have detected continuum in 78 objects and measured redshifts in 50 of them. We supplemented our redshift estimates with additional values taken from the literature (Rigopoulou et al. 2000; and Franceschini et al. 2003).

In Table 3 we give the redshifts and the emission line fluxes measured from the present spectra. In the majority of the spectra we detect [OII] $\lambda 3727$ and in some cases the [OIII] $\lambda 4958$ and $\lambda 5007$ lines as well. In half a dozen galaxies we measured $\mathrm{H}_{\beta} \lambda 4861$ line fluxes. Our measured redshifts are in agreement with the redshifts of Sawicki \& Mallen Ornellas (2003), although the two lists are complementary as these authors target lower redshift objects. Although we did not perform a detailed classification of objects based on their spectral properties, it is evident that the majority of the sample seem to be starburst galaxies in the redshift range $z=0.6-1.2$. We detected only a small fraction (less than $10 \%$ ) of elliptical galaxies ${ }^{2}$. The highest redshift object in our sample (No. 48) is a QSO at $z=$ 1.561. Because of its redshift the Balmer lines are redshifted into the near-infrared regime; in our optical spectra we detected C III] $\lambda 1909$ and MgII $\lambda 2798 \AA$. There is very good agreement between the redshift determined from the present optical spectra and the near-infrared spectra of Franceschini et al. (2003). Redshifts for objects Nos. 37 and 89 in our sample were determined independently from near-infrared data as well, and the agreement with our estimates is quite good. Based on the measured [OII] $\lambda 3727$ line fluxes we have calculated the individual expected SFR ([OII] 13727) (see Sect. 4, for details). We note that the spectral profiles were examined carefully in order to exclude objects displaying broad lines (i.e. containing type 1 Seyferts and QSOs) from the further estimates of the luminosity function (LF) and the total SFR density (see Sects. 5.1 and 5.2).

\subsection{Median redshift}

We estimated the median redshift as a function of the $I_{\mathrm{AB}}$ magnitude using the values given in Table 2. A plot of the median redshift vs. $I_{\mathrm{AB}}$ magnitude is presented in Fig. 3 . For the plot we have used the entire FORS sample, i.e. objects from the main WFPC2 area, the Flanking Fields and the ISO selected targets

2 We labeled as "ellipticals" those galaxies whose spectra show the absorption $\mathrm{CaII} \mathrm{H}$, K features.

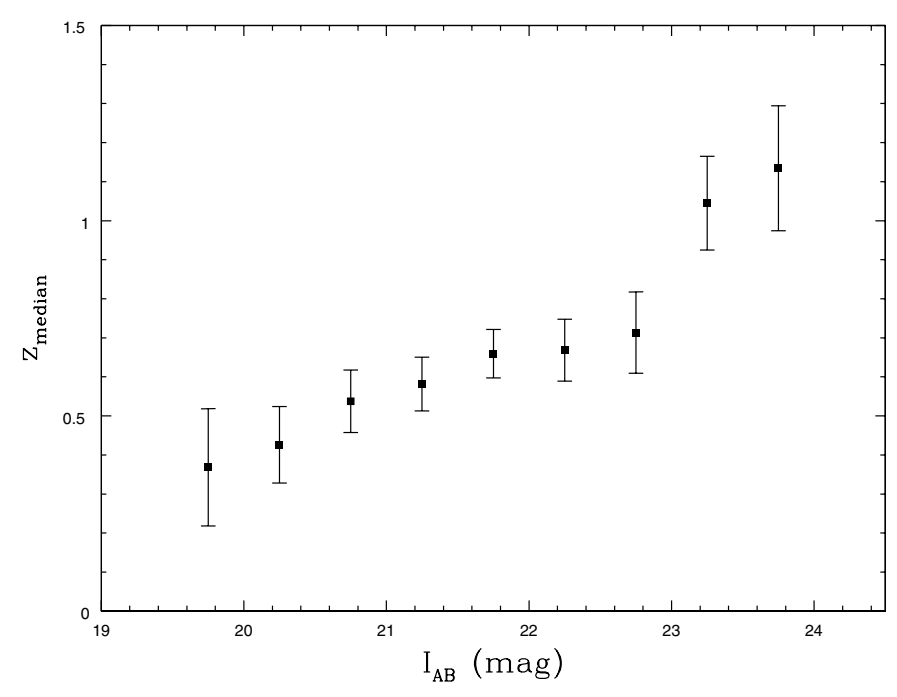

Fig. 3. Median redshift as a function of $I_{\mathrm{AB}}$.

(see Table 2). Although we have determined redshifts for the majority of the objects, the sample is rather incomplete at the fainter magnitudes. Assuming that $n_{i}(z)$ and $n_{i}($ tot $)$ are, respectively, the number of galaxies with spectroscopic redshift and the total number of galaxies (down to $I \sim 23.5$ ), then the completeness function in a given magnitude interval is defined as:

$\eta_{i}=n_{i}(z) / n_{i}(\mathrm{tot})$

In estimating the completeness function (Eq. (1)) we assumed that there are no biases or systematic effects and that the measured redshifts provide a fair sample of all possible redshifts in each bin. We then correct for the incompleteness by dividing the number of galaxies in each bin by $\eta_{i}$. In Fig. 3 we plotted the galaxy redshift as a function of $I_{\mathrm{AB}}$ magnitude for the entire FORS sample. We find that a median redshift of 1.16 is reached by $I_{\mathrm{AB}} \sim 23.5$ (corrected for incompleteness).

Finally, Fig. 4 shows the redshift distribution for our spectroscopic sample. The distribution shows an evident peak at $z \sim$ 0.58 which is most likely due to the large scale structure present in the HDF-S region (e.g. Arnouts et al. 2002; Vanzella et al. 2002).

\section{Extinction and SFR estimates}

For ionization-bounded HII regions the Balmer emission line luminosities scale directly with the ionizing luminosities of the exciting stars and thus are proportional to the star formation rate (SFR). Therefore, it is possible to use the Balmer emission lines to derive SFR in each galaxy. Traditionally, $\mathrm{H}_{\alpha}$ has been used to estimate SFR in galaxies in the redshift range up to $z \sim$ 0.4; beyond this redshift, $\mathrm{H}_{\alpha}$ moves out of the optical window and can be accessed at $z>0.8$ in the near-infrared regime. At redshifts $0.5<z<1.4$, the [OII] $\lambda 3727$ emission line can be used to study the star formation rates.

The conversion factor between [OII] luminosity and SFR is computed using an evolutionary synthesis model. To estimate SFR for the current sample of HDFS galaxies we use the relationship given by Kennicutt (1998) assuming a Salpeter IMF 


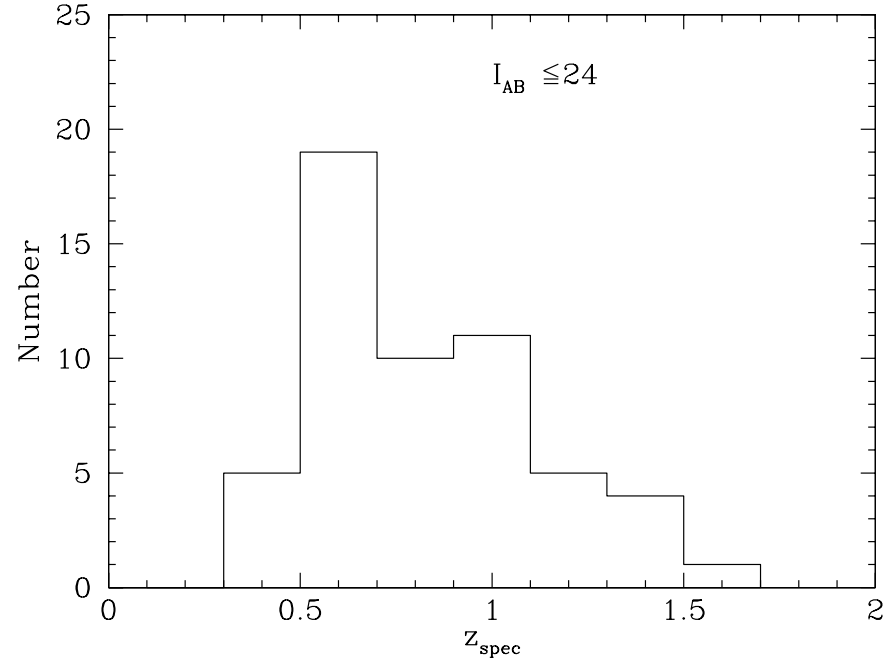

Fig. 4. Redshift distribution for all objects with confirmed spectroscopic redshifts.

(0.1-100 $\left.M_{\odot}\right)$, a constant star formation scenario and solar abundances:

$\operatorname{SFR}\left(M_{\odot} \mathrm{yr}^{-1}\right)=1.4 \times 10^{-41} L([\mathrm{OII}])\left(\mathrm{erg} \mathrm{s}^{-1}\right)$

[OII] $\lambda 3727$ luminosities were calculated from the measured [OII] $\lambda 3727$ fluxes and the measured redshifts. We applied aperture correction factors (with average value of 1.3) to account for the light missed by our $1.2^{\prime \prime}$ slit. We calculated aperture corrections in two different ways: (1) we smoothed the WFPC2 F814 image to a resolution of 1.2" (to simulate the seeing during the present observations) and then measured the ratio between the total object counts (in the image) and the object counts through an artificial 1.2" slit; (2) for each galaxy we estimated the continuum flux at $3727 \AA$ from the $V_{\mathrm{AB}}$ magnitude (recall that for $z \sim 0.6$ the $V$ band filter samples the restframe UV light) and calculated the [OII] $\lambda 3727$ flux from the Equivalent Width (EW, [OII] 33727 ) measured from our spectra. Both methods lead to similar correction factors of $\sim 1.3$.

However, because the observed [OII] $\lambda 3727$ line flux is extremely sensitive to extinction, an estimate of extinction should be made before we calculate SFRs. We base our extinction estimates on the comparison between predicted and observed $V-K$ colour index (magnitudes taken from Table 2). We use the Starburst99 code (Leitherer et al. 1999) for various star formation histories (i.e., bursts of different durations and ages, and continuous star formation) and calculate the range of intrinsic colours. The intrinsic $V-K$ colours predicted by the models are in the range 1.1-1.5. We apply infrared (Poggianti 1997) and optical (Coleman 1980) K-corrections for spiral (Sc) galaxies (assuming the median redshift of our sample $z \sim 0.6$ ). Comparing the observed $V-K$ colours of our galaxies with the predicted ones we obtain a median extinction $A_{\mathrm{V}}$ of 0.9 assuming a screen model for the extinction (we assume no extinction in the $K$-band). This corresponds to an upwards correction factor of 3.5 in the [OII] 33727 line fluxes. We list the final aperture-corrected and extinction-corrected individual SFRs in Table 3.

\section{5. [OII] 33727 luminosity function and global SFR density}

\section{1. [OII]ג3727 luminosity function}

A direct estimate of the present-day SFR activity in the Universe can be obtained by constructing the [OII] $\lambda 3727$ luminosity function (LF) from the present sample galaxies. We used the the $V / V_{\max }$ method (e.g. Felten 1977) to estimate the number density of galaxies in the various luminosity bins. In computing $V_{\max }$ however, we have to take into account that the sample is incomplete especially towards the fainter magnitude bins. Given the completeness function (Eq. (1)) estimated in Sect. 3.2 the appropriate formula for each galaxy's volume $V_{\max }$ is:

$V_{\max }=\int \eta_{i} \frac{\mathrm{d}^{2} V_{z}}{\mathrm{~d} \Omega \mathrm{d} z} \Delta \Omega \mathrm{d} z$

In the $V / V_{\max }$ method the luminosity density is obtained from:

$\phi(L) \Delta(L)=\sum_{i} \frac{1}{V_{\max }\left(L_{i}\right)}$

where $V_{\max }$ is the maximum volume over which a galaxy is observable given the survey's apparent luminosity and the selection criteria. The sum includes all galaxies in the luminosity interval $L_{i} \pm 0.5 \Delta L$. The errors are obtained assuming a Gaussian distribution which may not be very realistic for bins at the bright and faint ends where statistics are rather poor.

The LF constructed according to the $V_{\max }$ method is shown in Fig. 5. We then fit the LF with a Schechter (1976) function, and the best-fitting parameters (for a limiting [OII] $\lambda 3727$ flux of $1.02 \times 10^{-17} \mathrm{erg} \mathrm{cm}^{-2} \mathrm{~s}^{-1}$ ) are: $\alpha=-1.24 \pm 0.12, \phi^{*}=$ $10^{-2.56 \pm 0.08} \mathrm{Mpc}^{-3}, L^{*}=10^{42.33 \pm 0.07} \mathrm{erg} \mathrm{s}^{-1}$. The solid line in Fig. 5 represents the Schechter function for the LF.

Since the luminosity function is well fitted by a Schechter function with $\alpha \leq-2, \phi(L)$ can be integrated over the whole range of luminosities:

$L_{\mathrm{tot}}=\int L \phi(L) \mathrm{d} L=\phi^{*} L^{*} \Gamma(2+\alpha)$.

For the observed luminosities the total [OII] $\lambda 3727$ luminosity density is $L_{[\mathrm{OII}]}=10^{39.42 \pm 0.05} \mathrm{erg} \mathrm{s}^{-1} \mathrm{Mpc}^{-3}$ (for $0.5<z<$ $1.5,\langle z\rangle=0.8$ ). We note that for a Schechter function the luminosity density is dominated by $L_{*}$ galaxies, so galaxies outside the observed luminosity range do not introduce large errors as long as galaxies near $L_{*}$ are included and the faint end slope is $\alpha \leq 2$ (e.g. Tresse et al. 2001). A similar result for the total luminosity density is obtained by summing up $L[\mathrm{OII}] / V_{\max }$ for each galaxy. This gives the directly observed luminosity density $L_{[\mathrm{OII}]}=10^{39.12} \mathrm{erg} \mathrm{s}^{-1} \mathrm{Mpc}^{-3}$.

In addition to the total [OII] $\lambda 3727$ luminosity density estimate we divided our sample into three redshift bins at $\langle z\rangle=$ 0.4 and $\langle z\rangle=0.8$ and $\langle z\rangle=1.2$, while for each individual bin we estimated the [OII] $\lambda 3727$ luminosity density. For each individual bin we used the sum of the individual densities for each galaxy (as described above) to find: $10^{38.40 \pm 0.19} \mathrm{erg} \mathrm{s}^{-1} \mathrm{Mpc}^{-3}$ at $\langle z\rangle=0.4,10^{38.70 \pm 0.14} \mathrm{erg} \mathrm{s}^{-1} \mathrm{Mpc}^{-3}$ at $\langle z\rangle=0.8$, and $10^{38.90 \pm 0.22} \mathrm{erg} \mathrm{s}^{-1} \mathrm{Mpc}^{-3}$ at $\langle z\rangle=1.2$. The [OII] luminosity 


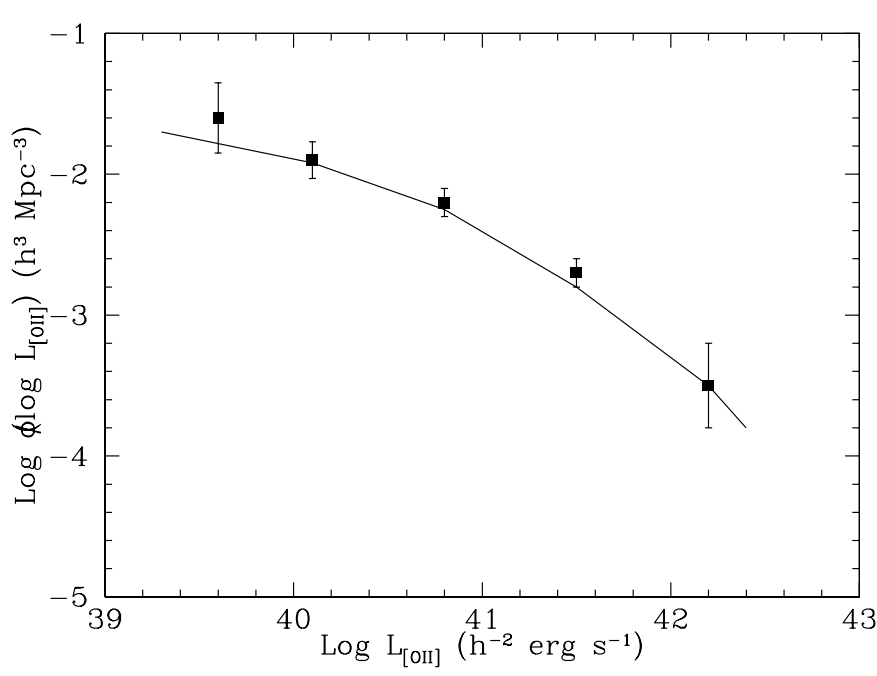

Fig. 5. [OII] luminosity function based on the $V_{\max }$ method with no extinction-corrections applied.

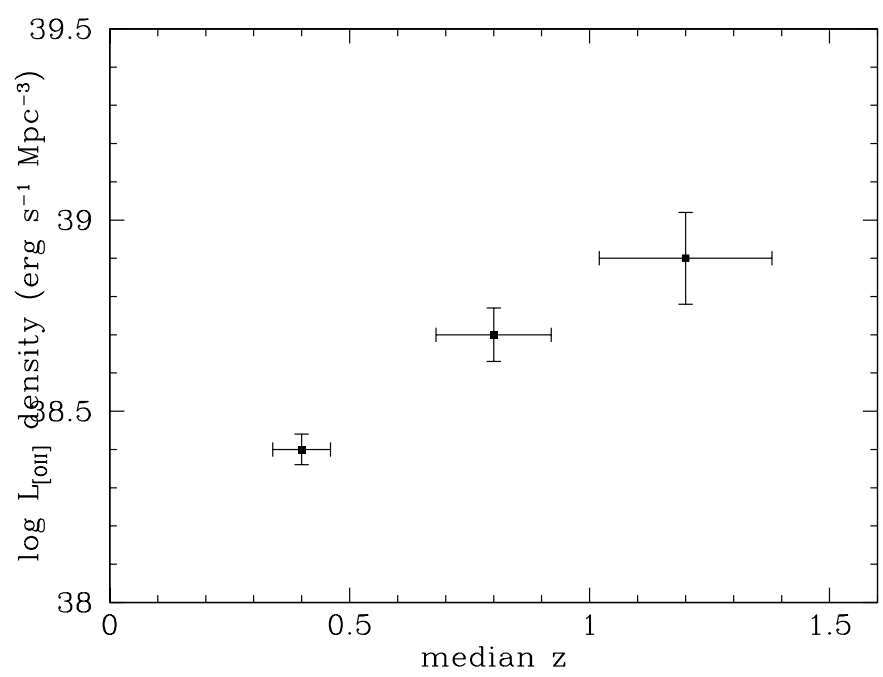

Fig. 6. $[\mathrm{OII}]$ luminosity density as a function of median redshift.

density as a function of median redshift is shown in Fig. 6. Although these later estimates suffer from small number statistics (in each bin we have $10<n<20$ galaxies), we note an evident trend towards higher luminosity density with increasing redshift over the range $0.4<z<1.2$.

\subsection{Global SFR density}

Using Eq. (2) we can transform L[OII] $] 3727$ into a global SFR density. The total [OII] $\lambda 3727$ luminosity density we estimated in 5.1, translates into an SFR density of $0.015 \pm$ $0.03 M_{\odot} \mathrm{yr}^{-1} \mathrm{Mpc}^{-3}$. If we assume a median extinction of $A_{\mathrm{V}}=0.9$ (see Sect. 4) then the total extinction-corrected [OII] $\lambda 3727$ luminosity density translates into SFR density of $0.11 \pm 0.02 M_{\odot} \mathrm{yr}^{-1} \mathrm{Mpc}^{-3}$.

In Fig. 7 we plot SFR densities vs. redshifts from the present spectroscopic survey (for the redshift range 0.4-1.2) supplemented with data from other surveys in the literature. We restricted ourselves to SFR estimates based on [OII] $\lambda 3727$ surveys in order to avoid conversions from $\mathrm{H}_{\alpha}$ to [OII] $\lambda 3727$

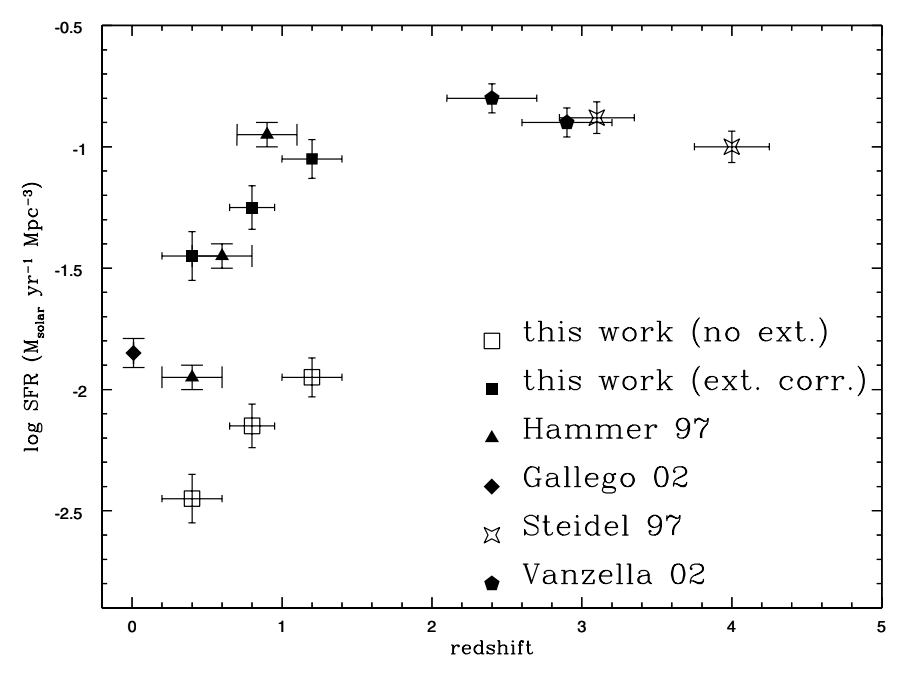

Fig. 7. SFR densities based on the present data, supplemented with literature values. Both the observed and extinction-corrected values are shown for the present sample and are represented as open and filled squares. Filled diamonds correspond to Gallego et al. (2002); filled triangles are for Hammer et al. (1997), filled pentagons for Vanzella et al. (2002) and filled stars are for Steidel et al. (1997).

luminosities. For the local Universe we used the SFR estimated by Gallego et al. (2002), and Hammer et al. (1997) for redshifts between 0.5 and 1.0. For redshifts beyond $z \simeq 1.5$ we used the results from Vanzella et al. (2002) and Steidel et al. (1997). Our results are in good agreement with the Canada-France-Redshift-Survey (CFRS, Hammer et al. 1997) results for the same redshift range. Unfortunately, most surveys provide only the observed luminosities and not the extinction corrected values. For the present data we plot both observed and extinction-corrected values.

Our present data show evidence for strong evolution from the local Universe out to $z \simeq 1.2$. Such a strong evolution of the SFR has also been observed with the $\mathrm{H}_{\alpha}$ survey of Tresse et al. (2002) and the CFRS (Hammer et al. 1997). Assuming that the evolution of the SFR density with redshift follows a relationship of the $(1+z)^{n}$ form, then the combination of all the SFR data points implies a value for $n \simeq 4$. We caution, though, that this result is to some degree dependent on the local SFR density value used. As an example, the local Universe SFR density value derived from the $\mathrm{H}_{\alpha}$ survey of Singleton et al. (2001) is lower than the value of Gallego et al. (1995). Irrespective of the local value, however, our present data show a clear rise in the SFR density by a factor of 6 in the $0.4<z<$ 1.2 regime.

\section{Conclusions}

We have presented new spectroscopic redshifts for 50 galaxies in the HDFS and Flanking Fields. We determined redshifts based on the detections of several emission lines such as [OII] $\lambda 3727, \mathrm{H}_{\beta}$ and [OIII] $\lambda 5007$ and the absorption features CaII H, K $\lambda \lambda 3968.5$ and $3933.7 \dot{A}$.

The redshift range of the present galaxy sample is $0.6<z<$ 1.2 with a median redshift of 1.13 (at $I \simeq 23.5$ not corrected for completeness). A large fraction of the galaxies detected are 
starbursts while the remaining $\sim 10 \%$ are ellipticals. The individual star formation rates have been estimated for the emission line objects and the extinction correction rates range between $0.5-30 M_{\odot} \mathrm{yr}^{-1}$. Based on the present data we have estimated the global SFR density of the Universe out to $z \sim 1.3$ and found it to be $0.11 \pm 0.02 M_{\odot} \mathrm{yr}^{-1} \mathrm{Mpc}^{-3}$ using the total extinction corrected [OII] 33727 luminosity density.

Our data show evidence of a strong evolution from the local $(z=0)$ Universe out to $z \sim 1.3$, following $(1+z)^{4}$. Finally, the availability of $8 \mathrm{~m}$ class telescopes allows us to sample the evolution of the $z \simeq 1$ Universe precisely and improve on previous determinations.

Acknowledgements. We acknowledge support of the EU TMR Network "Probing the Origin of the Extragalactic Background Radiation" (HPRN-CT-2000-00138). We thank Michel Dennefeld and his team for acquiring the data and Niranjan Thatte for help with data analysis. S.B. thanks MPE for the hospitality during his visits.

\section{References}

Appenzeller, I., Bender, R., Boehnhardt, H., et al. 2000, ESO Messenger, 100, 44

Arnouts, S., Moscardini, L., Vanzella, E., et al. 2002, MNRAS, 329, 355

Bergeron, J., Petitjean, P., Cristiani, S., et al. 1999, A\&A, 343, 40 Casertano, S., de Mello, D., Dickinson, M., et al. 2000, AJ, 120, 2747

Cohen, J. G., Hogg, D. W., Blandford, R., et al. 2000, ApJ, 538, 29

Coleman, G. D., Wu, C.-C., \& Weedman, D. W. 1980, ApJ, 43, 393
Cristiani, S., Appenzeller, I., Arnouts, S., et al. 2000, A\&A, 359, 489

Da Costa, L., Nonino, M., Rengelink, R., et al. 1998, A\&A, submitted [arXiv: astroph/9812105]

Felten, J. E. 1977, AJ, 82, 861

Franceschini, A., Berta, S., Rigopoulou, D., et al. 2003, A\&A, 403, 501

Gallego, J., Zamorano, J., Aragon-Salamanca, A., \& Rego, M. 1995, ApJ, 455, L1

Gallego, J., Garcia-Dabo, C. E., Zamorano, J., Aragon-Salamanca, A., \& Rego, M. 2002, ApJ, 570, L1

Hammer, F., Flores, H., \& Lilly, S. J. 1997, ApJ, 481, 49

Kennicutt, R. C. 1998, ARA\&A, 36, 189

Leitherer, C., Schaerer, D., Goldader, J. D., et al. 1999, ApJS, 123, 3

Mann, R. G., Oliver, S., Carballo, R., et al. 2002, MNRAS, 332, 549

Oliver, S., Mann, R. G., Carballo, R., et al. 2002, MNRAS, 332, 536

Page, M. J., \& Carrera, F. J. 2000, MNRAS, 311, 433

Poggianti, B. M. 1997, A\&AS, 122, 399

Rigopoulou, D., Franceschini, A., Aussel, H., et al. 2000, ApJ, 537, L85

Rudnick, G., Franx, M., Rix, H.-W., et al. 2001, AJ, 122, 2205

Saracco, P., Giallongo, E., Cristiani, S., et al. 2001, A\&A, 375, 1

Sawicki, M., \& Mallen-Ornellas, G. 2003, AJ, 126, 1208

Schechter, P. 1976, ApJ, 203, 297

Singleton, C. 2001, Ph.D. Thesis, Univ. Nottingham

Teplitz, H. I., Hill, R. S., Malumuth, E. M., et al. 2001, ApJ, 548, 127

Tresse, L., Dennefeld, M., Petitjean, P., et al. 1999, A\&A, 346, 21

Vanzella, E., Cristiani, S., Saracco, P., et al. 2001, AJ, 122, 2190

Vanzella, E., Cristiani, S., Arnouts, S., et al. 2002, A\&A, 396, 847

Williams, R. E., Baum, S., Bergeron, L. E., et al. 2000, AJ, 120, 2735 
D. Rigopoulou et al.: FORS spectra of HDFS galaxies, Online Material $p 1$

\section{Online Material}


D. Rigopoulou et al.: FORS spectra of HDFS galaxies, Online Material p 2
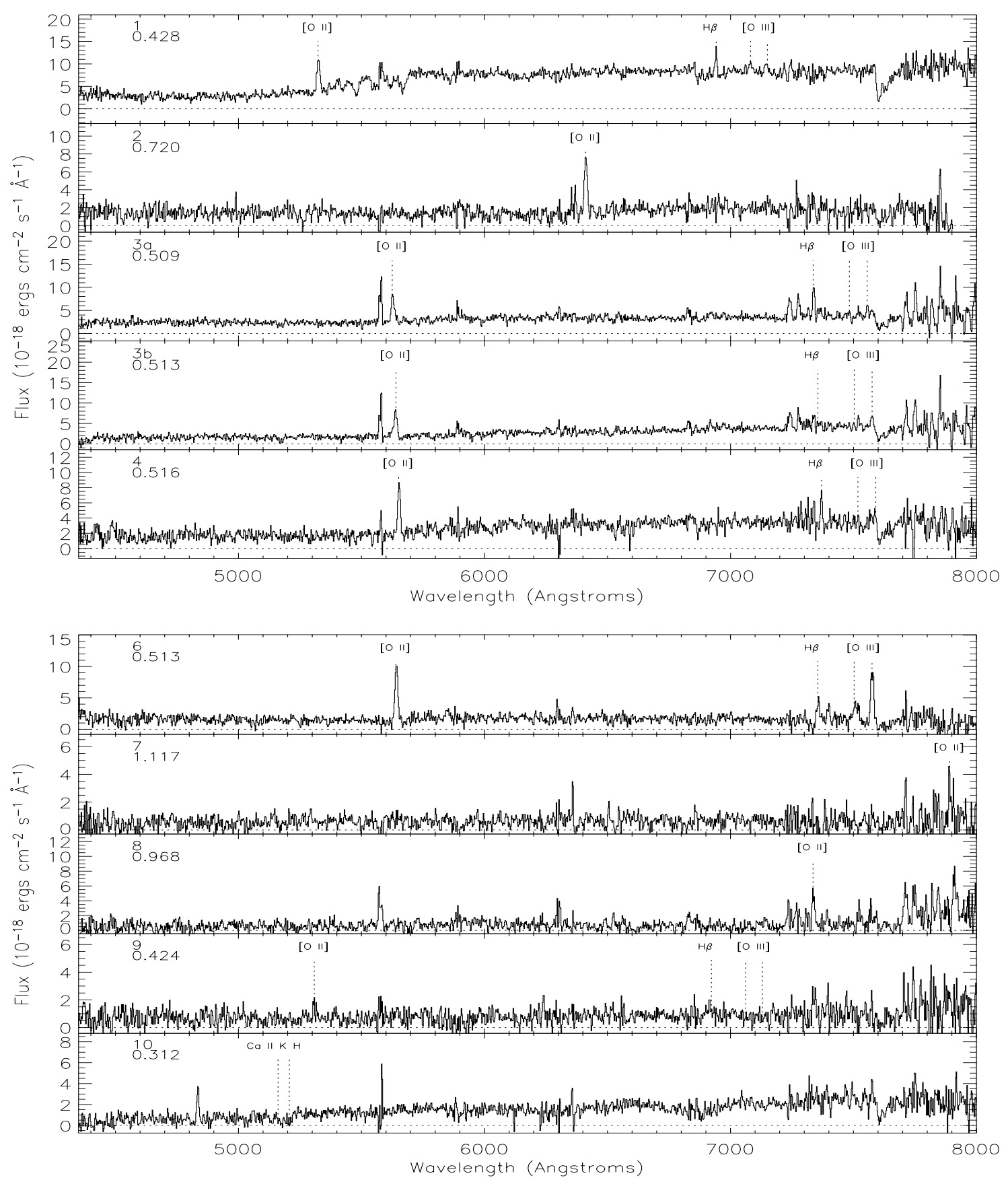

(a)

Fig. 1. Spectra of galaxies observed in the HDFS. The ordinate gives the flux (in erg $\mathrm{cm}^{-2} \mathrm{~s}^{-1} \AA^{-1}$ ). The wavelength coverage extends between $4200 \AA$ and $8200 \AA$. The numbers on the top left corner of the spectra correspond to objects from Table 2 while the second number denotes the redshift. Note that for objects 1-39 the resolution is $\Delta \lambda / \lambda \sim 440$ while for objects 40 to 94 resolution is $\Delta \lambda / \lambda \sim 260$ (due to different grism filter combination). Since the wavelength coverage is common we present both spectra together. 
D. Rigopoulou et al.: FORS spectra of HDFS galaxies, Online Material p 3
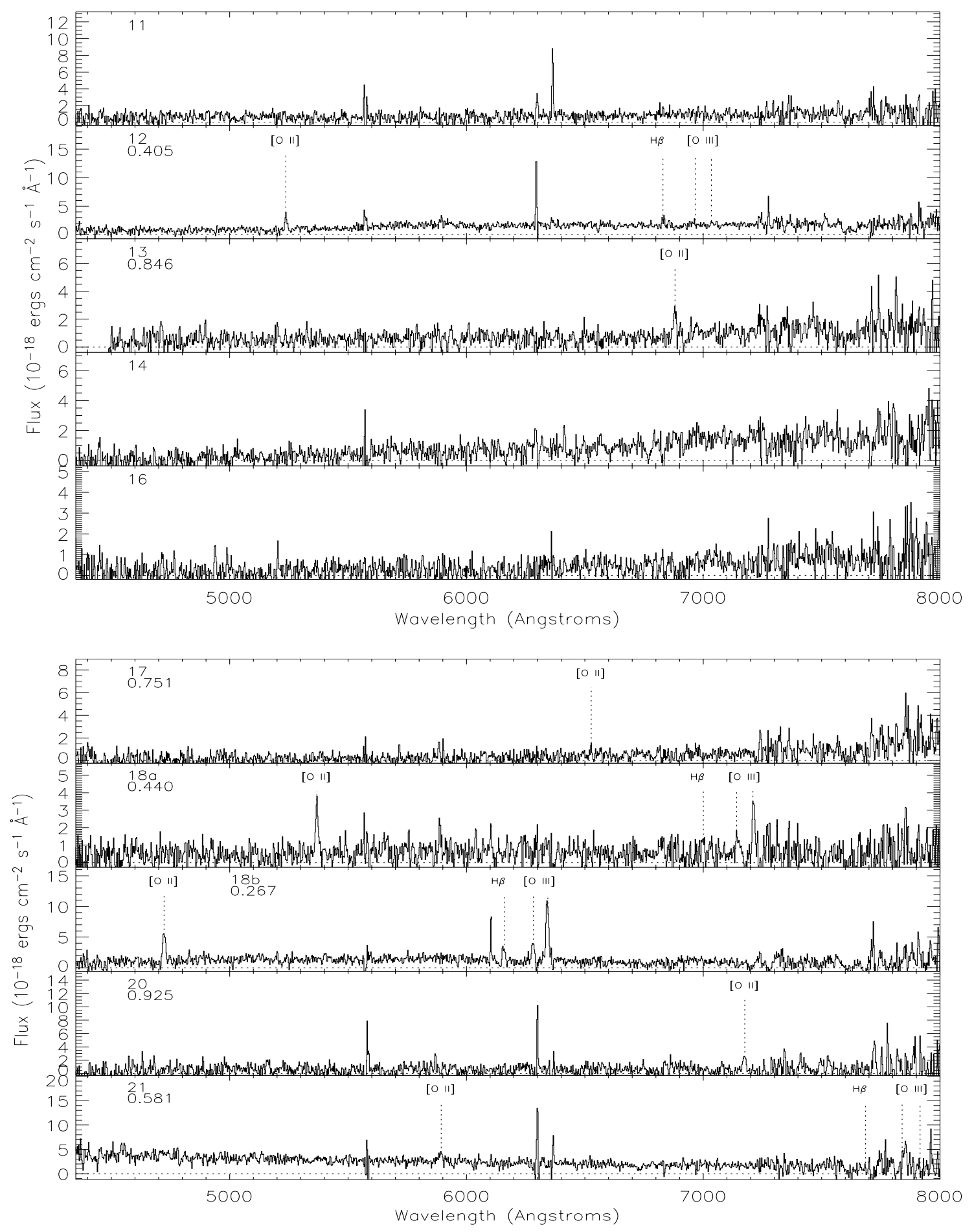

(b)

Fig. 1. continued. 
D. Rigopoulou et al.: FORS spectra of HDFS galaxies, Online Material p 4
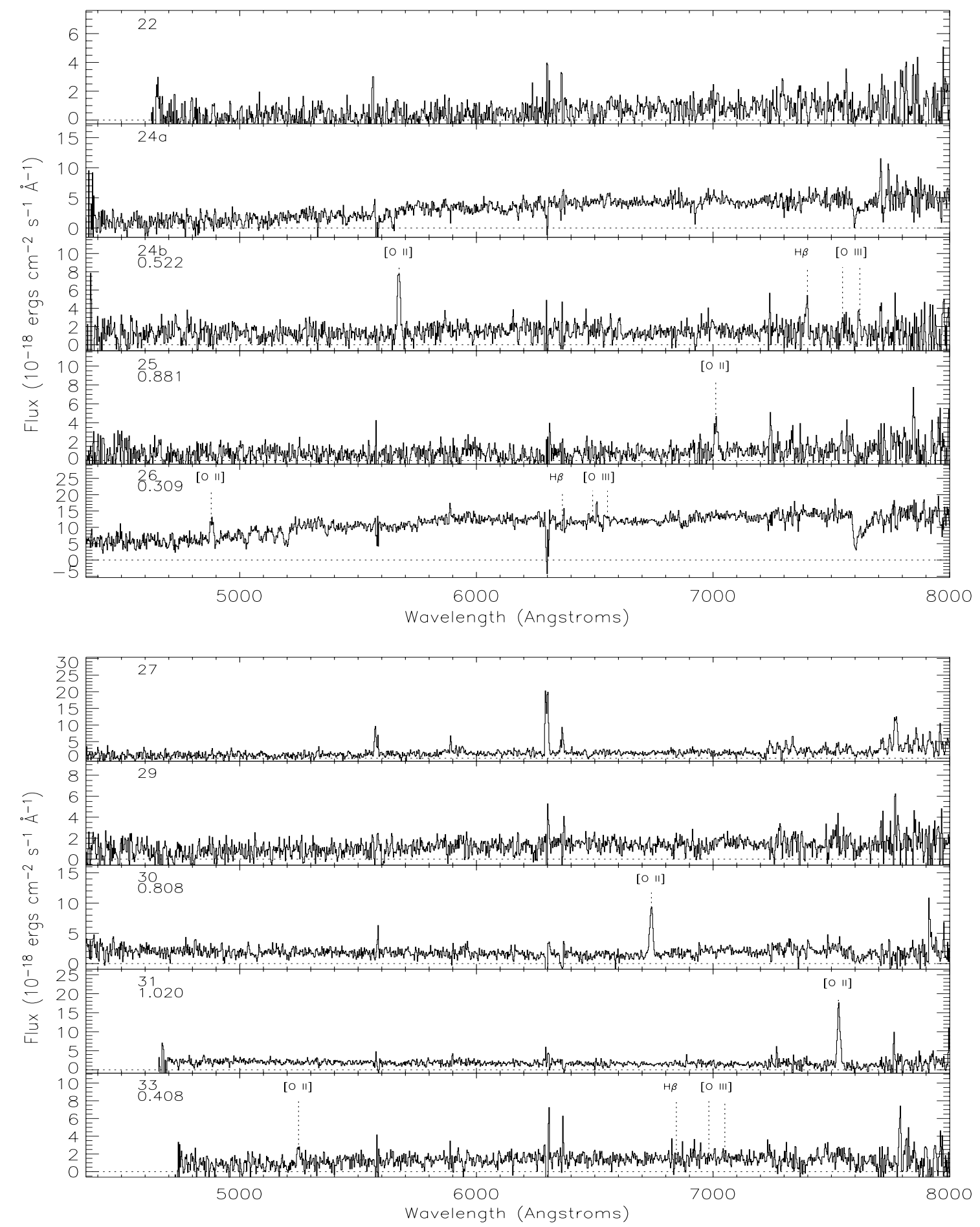

(c)

Fig. 1. continued. 
D. Rigopoulou et al.: FORS spectra of HDFS galaxies, Online Material p 5


(d)

Fig. 1. continued. 
D. Rigopoulou et al.: FORS spectra of HDFS galaxies, Online Material p 6
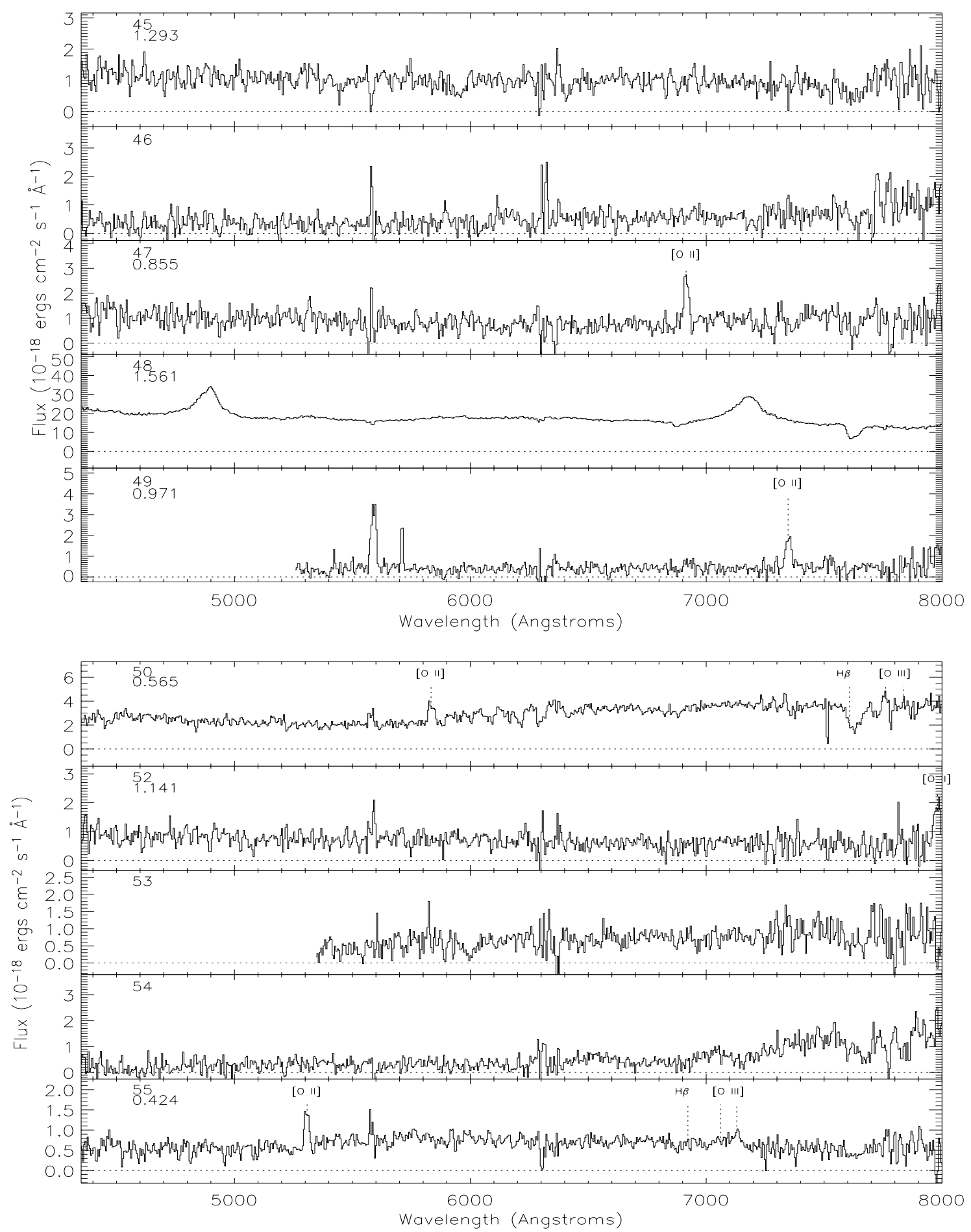

(e)

Fig. 1. continued. 
D. Rigopoulou et al.: FORS spectra of HDFS galaxies, Online Material $p 7$
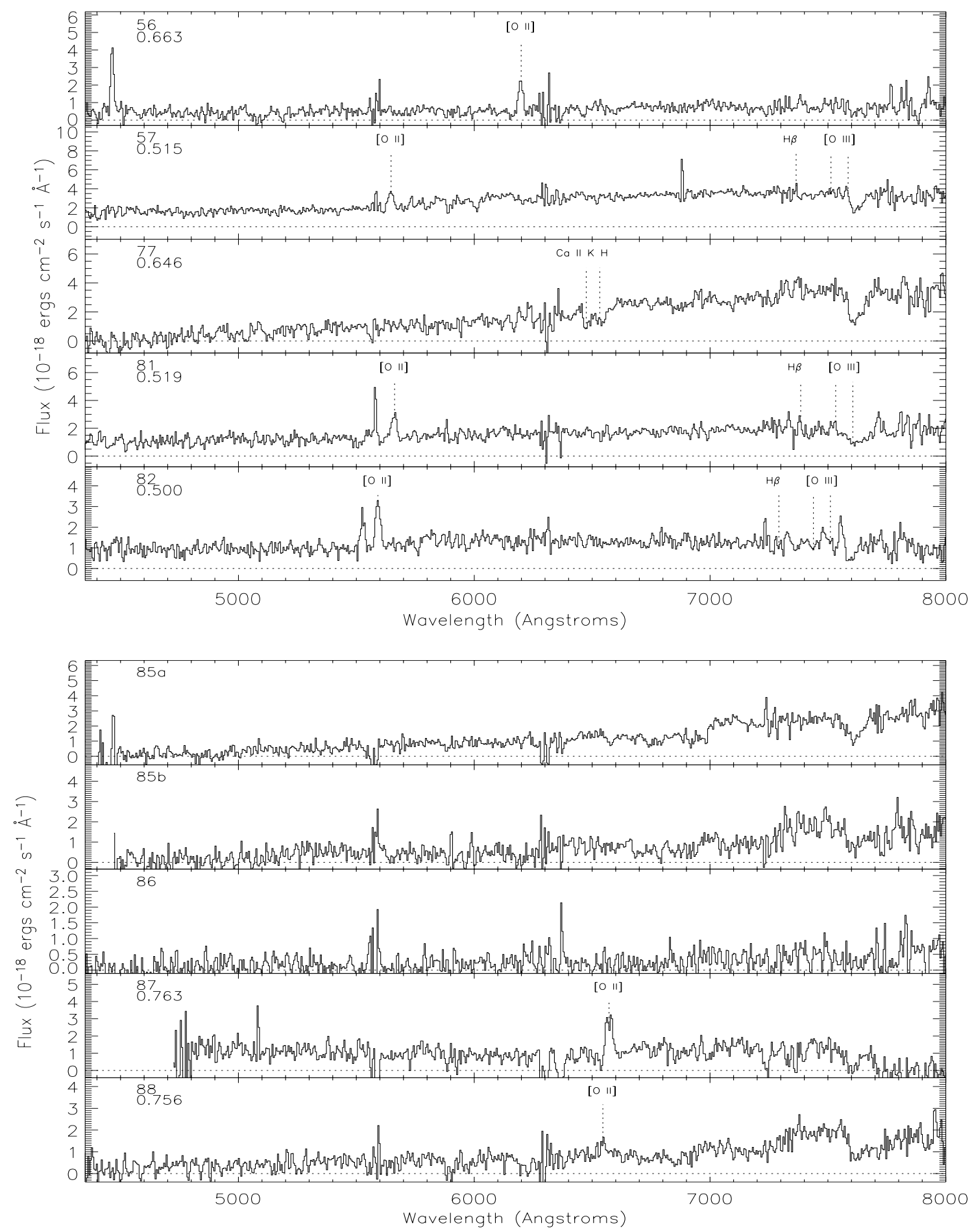

(f)

Fig. 1. continued. 
D. Rigopoulou et al.: FORS spectra of HDFS galaxies, Online Material $p 8$

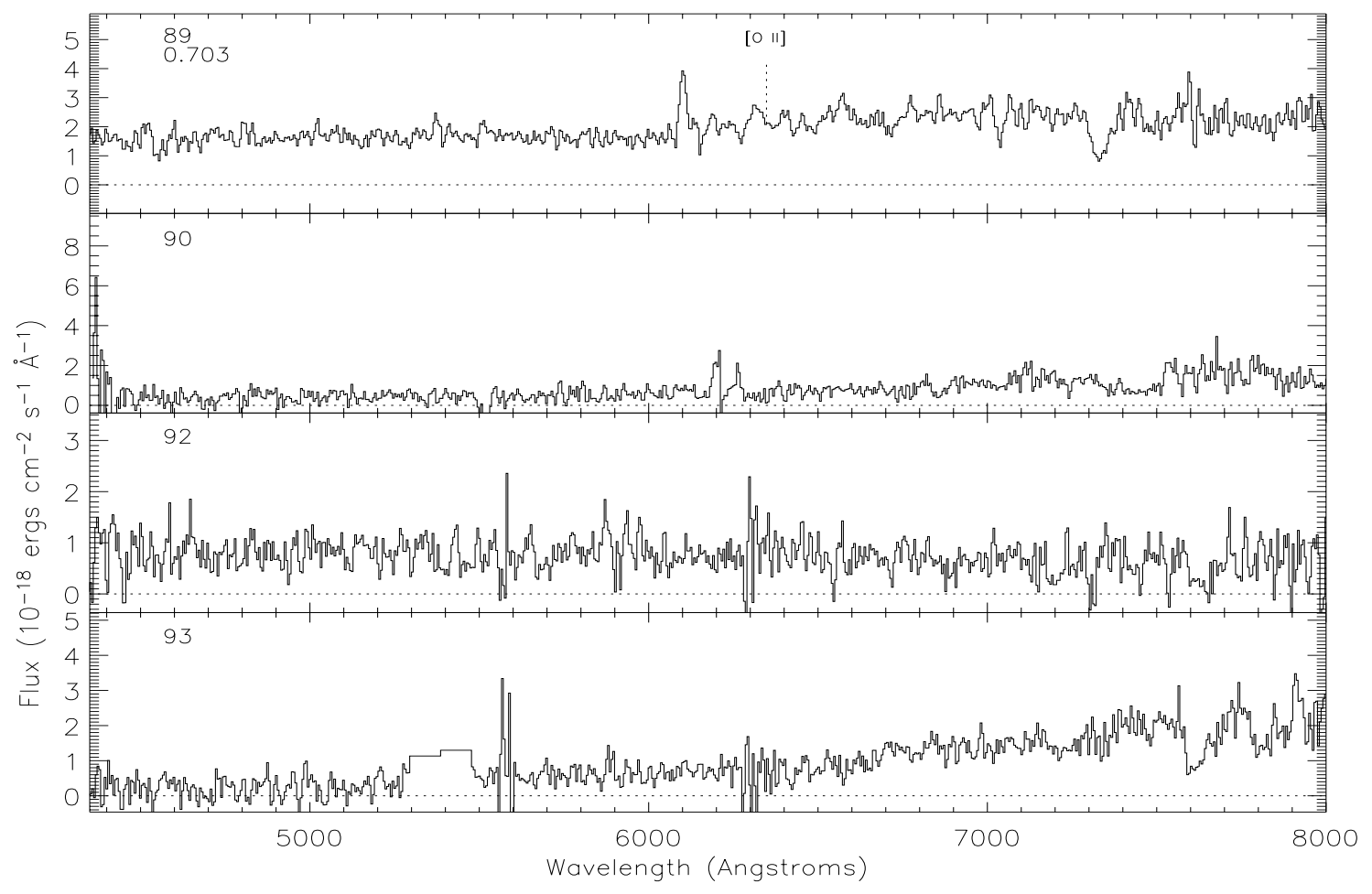

(g)

Fig. 1. continued. 
D. Rigopoulou et al.: FORS spectra of HDFS galaxies, Online Material $p 9$
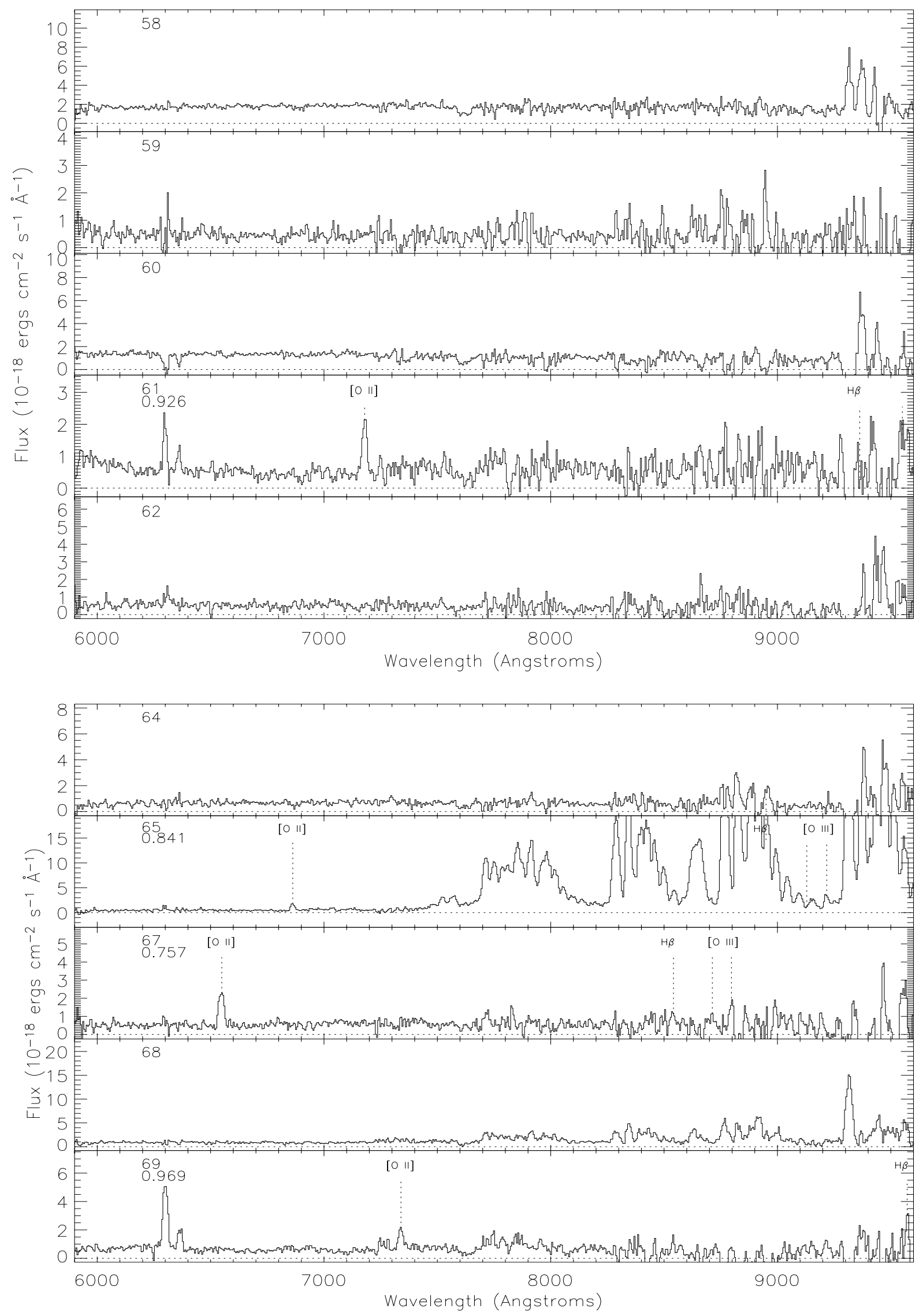

(a)

Fig. 2. Same as Fig. 1. The wavelength coverage extends between 6000-9200 Å. 
D. Rigopoulou et al.: FORS spectra of HDFS galaxies, Online Material p 10

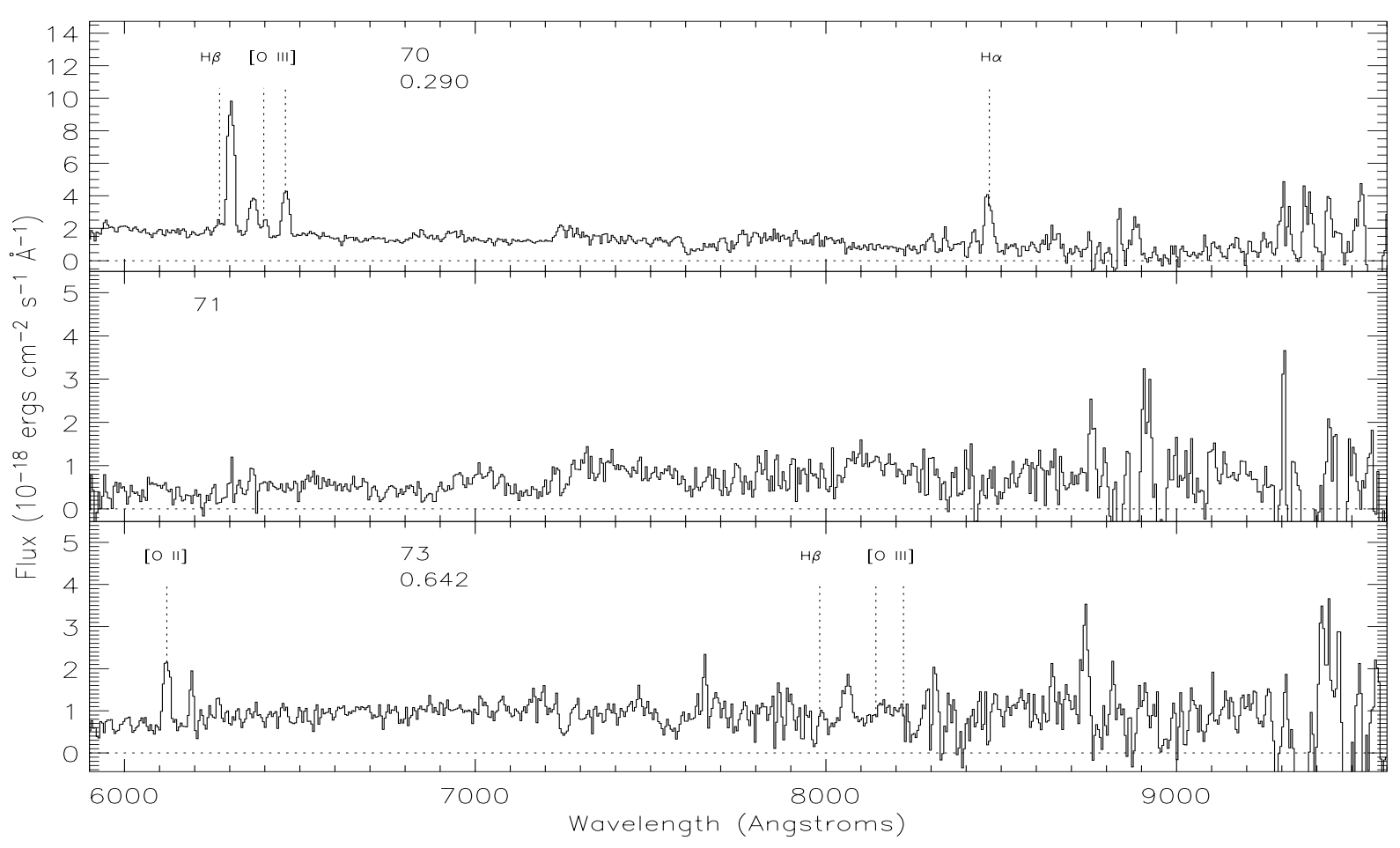

(b)

Fig. 2. continued. 